\title{
Análise da produtividade científica dos docentes da Universidade Estadual Paulista, campus de Marília/SP
}

\section{Rondinelli Donizetti Herculano}

Departamento de Ciências Biológicas, FCLAs, Universidade Estadual Paulista, Assis (Brasil)

Ana Maria Q. Norberto

Fonoaudióloga, Mestranda em Neurociências pelo Departamento de Neurociências e Ciências do Comportamento, FMRP, Universidade de São Paulo, Ribeirão Preto (Brasil)

O objetivo do presente trabalho foi analisar/quantificar a produtividade científica dos docentes pertencentes à Universidade de Estadual Paulista (UNESP), campus de Marília/SP. Para cada professor, analisamos o número total de artigos na plataforma Lattes, no ISI Web of Science e no SCOPUS. Além disso, verificamos o total de livros publicados, capítulos de livros publicados e orientações em Programas de Pós-Graduação (PPG). A análise dos nove departamentos da Faculdade de Filosofia e Ciências (FFC) mostrou que o Departamento de Ciências Políticas e Econômicas possui uma média elevada $(21,42)$ de artigos na Plataforma Lattes, quando comparado aos outros departamentos. Entretanto, o Departamento de Fonoaudiologia apresentou médias mais altas em artigos indexados pelo ISI $(2,61)$ e SCOPUS $(4,39)$, bem como o índice- $h$, nessas plataformas. Em relação aos programas de pós-graduação, o Departamento de Administração e Supervisão Escolar foi o que apresentou a maior média de orientações em andamento e/ou concluídas. $O$ desempenho mais baixo, em relação aos artigos publicados pelo ISI e SCOPUS, pelos departamentos na área de Humanas/Humanidades pode estar associado às características do campo da concentração e da cobertura limitada do banco de dados ISI e SCOPUS, indicando que o índice-h é muito sensível ao campo de concentração.

Palavras-chave: Cienciometria; Indicadores de produção científica; Bibliometria. 


\section{Scientific research output evaluation of professors of Sao Paulo State University, Marília/SP}

In this work, an analysis of scientific bibliographic productivity was made using the Faculdade de Filosofia e Ciências, Universidade Estadual Paulista (FFC-UNESP) as example. It is composed by nine departments which offer altogether nine undergraduate courses: 1) Archival, 2) Library, 3) Speech Therapy, 4) Pedagogy, 5) International Relations, 6) Physiotherapy, 7) Occupational Therapy, 8) Philosophy, 9) Social Sciences and six graduate programs leading to M.S. and Ph.D. degrees. Moreover, when analyzing the different courses of FFC-UNESP, they represent typical academic organization in Brazil and Latin America and could be taken as a model for analyzing other Brazilian research institutions. Using data retrieved from the Lattes Plataform database (Curriculum Lattes) we have quantitatively the scientific productivity percentage of professors at UNESP. We observed that bibliometric evaluations using the Curriculum Lattes (CL) showed that the professors published papers in journal are not indexed by ISI and SCOPUS. This analysis was made using: 1) the total number of papers (indexed in Curriculum Lattes database), 2) the number of papers indexed by Thomson ISI Web of Science database and SCOPUS database, and 3) the Hirsch (h-index) by ISI and SCOPUS. Bibliometric evaluations of departments showed a better performance of Political Science and Economics Department when compared to others departments, in relation total number of papers (indexed in Curriculum Lattes database). We also analyzed the academic advisory (Master's Thesis and Ph.D. Thesis) by nine departments of FFC/UNESP. The Administration and School Supervision Department presented a higher academic advisory (concluded and current) when compared to the others departments.

Keywords: Periodical index; Scientific publication indicators; Scientometrics.

\section{Introdução}

Toda e qualquer ciência deve ser avaliada periodicamente, a fim de mostrar à sociedade sua importância e seus avanços, dando, desta forma, 
um retorno do investimento que nela foi aplicado. Segundo Price (1965), quando um homem trabalha e produz alguma coisa nova e o resultado é uma publicação, então ele está fazendo ciência.

De acordo com Vessuri (1987), a ciência que não é publicada não existe, além disso, Macias-Chapula (1998) ressalta que publicar os resultados de suas pesquisas é um compromisso que os cientistas são compelidos a cumprir (MERTON, 1957).

O avanço do conhecimento produzido pelos pesquisadores tem que ser transformado em informação acessível para a comunidade científica. Portanto, a pesquisa é desenvolvida em um contexto de troca. A publicação dos resultados de pesquisa tem três objetivos: divulgar descobertas científicas, salvaguardar a propriedade intelectual e alcançar a fama (OKUBO, 1997).

A publicação é, para a maioria daqueles que atuam na pesquisa, não só um indicador, mas o produto final de todo um esforço criativo. Dessa forma, qualquer contribuição só é reconhecida após ser publicada, julgada e incorporada, de alguma maneira, aos conhecimentos já existentes. O ciclo do conhecimento só se completa após a aceitação da descoberta por outros cientistas da mesma área e isso se dá através da publicação. Por esse motivo, é importante avaliar uma área do conhecimento, seguindo os passos da publicação científica de um ou vários pesquisadores de determinado ramo da ciência (BRAGA, 1974).

Dentre os métodos quantitativos utilizados para medir e avaliar o conhecimento científico encontram-se a bibliometria, a cienciometria e a informetria. Cada um deles destina-se a medir, sob enfoques distintos, aspectos específicos de um corpus de conhecimento.

De acordo com Price (1976), os indicadores cienciométricos podem ajudar a mensurar a contribuição dada por cada país ao acervo mundial de pesquisa, além de localizar geograficamente as pesquisas por autor, assunto e acompanhar o crescimento em cada área da ciência. A cienciometria é todo tipo de análise quantitativa da ciência, baseada em fontes secundárias, sem observação direta do processo de produção de conhecimento e sem avaliação direta dos resultados produzidos (LEA VELHO, 1996).

Para orientar as políticas específicas de determinado setor e justificar a atividade científica, recorre-se a métodos quantitativos e qualitativos de avaliação. Assim, possibilita-se medir os níveis de desenvolvimento alcançados por uma disciplina $e$ as taxas de produtividade dos pesquisadores envolvidos, detectar instituições e cursos com maior potencial de crescimento e determinar de escalas de prioridades para a distribuição de recursos (VANTI, 2006).

Indicadores científicos são cada vez mais necessários para os que formulam as políticas científicas no plano nacional, os quais devem determinar as prioridades de pesquisa entre e dentro dos mais diversos campos científicos. Os tomadores de decisões, que pertencem tanto aos órgãos de governo quanto às agências financiadoras, precisam de dados sistemáticos sobre o desempenho das diversas áreas para poder escolher, 
com melhores fundamentos, nas quais concentrar os recursos financeiros e humanos limitados de que dispõem (IRVINE; MARTIN, 1989).

A análise da produtividade cientifica é sensível à área de atuação, ou seja, claramente, cada área tem suas particularidades. De acordo com Price (1965), "cada área tem sua própria maquinaria para manejar os processos de publicação e comunicação entre pessoas". As áreas duras publicam muito no exterior e dão preferência a trabalhos curtos. No outro extremo, estão as áreas sociais, publicando para um leitor brasileiro e dando relativamente mais preferência aos trabalhos longos (CASTRO, 1985).

Segundo Spinak (1996), as ferramentas utilizadas pelos indexadores internacionais selecionam revistas, com certa parcialidade, e nem sempre são adequadas para avaliar Ciência e Tecnologia dos países periféricos. Este mesmo autor observa que, em qualquer campo da ciência, os artigos se concentram nas mesmas revistas multidisciplinares de alto impacto, que os cobrem como "caudas de cometas", assim como cerca de sete mil revistas indexadas no ISI cobrem mais que suficientemente $90 \%$ da literatura que realmente tem valor no meio acadêmico.

De acordo com Herculano (2009a), outra forma de verificar a quantidade e/ou título de periódicos é por meio do sistema de currículos chamado Plataforma Lattes (PLATAFORMA LATTES, 2011). Este banco de dados inclui currículos dos pesquisadores/professores pertencentes às instituições acadêmicas brasileiras. A plataforma Lattes possui cerca de 850 mil currículos, sendo que 30\% destes são de mestres e doutores e $38 \%$ de graduados e estudantes de graduação, que pertencem à cerca de 4000 diferentes instituições de pesquisa registradas no sistema.

Além da plataforma Lattes, existem outros renomados bancos de dados internacionais, tais como: Thompson ISI Web of Science (THOMPSON ISI WEB of SCIENCE, 2011), SCOPUS (SCOPUS-ELSEVIER, 2011) e o SCielo (SCIELO, 2011). A base de dados ISI (GARFIELD, 1995) representa um conjunto de periódicos que constituem uma literatura influente internacionalmente. A mesma representa a porção de pesquisa que é publicada e citada na literatura da elite internacional. Além disso, geralmente representa a melhor ciência realizada em qualquer nação. No entanto, sabe-se que pesquisas de ponta são também publicadas em periódicos locais ou regionais não indexados no ISI.

Recentemente, o "SCOPUS" (SCOPUS-ELSEVIER, 2011), que é o maior banco de dados de resumos e citações, com cerca de 17 mil revistas, surgiu para competir com Thompson ISI Web of Science, que possui uma cobertura pequena, principalmente porque somente uma pequena porcentagem (5\%) dos artigos publicados no mundo está incluída no ISI, ou seja, cerca de 7 mil títulos.

Recentemente, alguns trabalhos relacionados à cienciometria (HERCULANO et al., 2008; 2009b; ROEDIGER, 2006; GLÄNZEL, 2006; STREHL, 2002; PACKER, 2006) foram publicados. Entretanto, não existem trabalhos detalhados referentes à publicação dos professores da UNESP. Considerando a importância da produção cientifica para as agências de fomento para liberação de verbas, cargos administrativos dentro das 
universidades, dentre outros, analisamos o perfil de publicação dos docentes pertencentes à Faculdade de Filosofia e Ciências, nos referidos bancos de dados.

\section{Métodos}

A coleta de dados foi realizada por meio de busca online na Internet, entre janeiro e fevereiro de 2011. O período de cobertura da pesquisa foi de 1976 até fevereiro de 2011. Primeiramente, houve o acesso ao site da FFC (PRICE, 1965) e seus respectivos departamentos. Nesta etapa, foi realizada a coleta de dados relativa ao total de docentes pertencentes aos nove cursos de graduação e seis programas de Pós-graduação do campus (FACULDADE DE FILOSOFIA E CIÊNCIAS, 2010), inclusive o recém-criado programa de Pós-Graduação em Fonoaudiologia (FACULDADE DE FILOSOFIA E CIÊNCIAS, 2011).

As informações relacionadas à quantidade de artigos, livros publicados, capítulos de livros e orientações em Programas de PósGraduação foram obtidas com base nas informações contidas no Currículo Lattes (CL) de cada docente. A busca foi realizada, acessando-se a "Plataforma Lattes", no campo "busca pesquisadores", e empregado o modo de busca simples. Digitou-se o nome completo de cada docente pertencente a cada departamento da unidade participante desta pesquisa (FFC).

A partir dos dados obtidos pelo currículo Lattes, acessou-se "Thompson ISI Web of Science", no campo "Advanced Search", para verificar se os periódicos publicados pelos docentes dos departamentos analisados estão indexados no ISI.

Da mesma forma, acessou-se "SCOPUS", no campo "Search", para verificar se os periódicos publicados pelos docentes dos departamentos analisados estão indexados no SCOPUS.

Para uma análise da relação tipo de publicação/índice- $h$, o mesmo foi calculado utilizando-se a fórmula de Hirsch, publicada em 2005 (HIRSCH, 2005), a qual estabelece que $h$ seja definido como o número de artigos publicados por um pesquisador, com citações maiores ou iguais a esse número. Este método tem a vantagem de avaliar a produção científica de pesquisadores porque combina produtividade e o impacto da pesquisa.

\section{Resultados}

O principal objetivo deste trabalho foi analisar a produtividade científica dos docentes da FFC-UNESP. Para isso, buscou-se os departamentos existentes na referida instituição e os programas de pósgraduação que fazem parte dela. Na Tabela 1, são apresentados os cursos de graduação, Programas de Pós-Graduação e número de docentes por departamentos da FFC-UNESP. 
Tabela 1 - Cursos de graduação e Programas de Pós-Graduação e números de docentes por departamentos de ensino da FFC - UNESP

\begin{tabular}{lccc}
\hline \multicolumn{1}{c}{ Departamento } & Cursos de Graduação & $\begin{array}{c}\text { Programas de Pós- } \\
\text { Graduação }\end{array}$ & $\begin{array}{c}\text { Número de } \\
\text { docentes }\end{array}$ \\
\hline $\begin{array}{l}\text { Psicologia da } \\
\text { Educação }\end{array}$ & Pedagogia & ------ & 11 \\
\hline Ciência da Informação & $\begin{array}{c}\text { Arquivologia e } \\
\text { Biblioteconomia }\end{array}$ & $\begin{array}{c}\text { Ciência da } \\
\text { Informação }\end{array}$ & 21 \\
\hline $\begin{array}{l}\text { Sociologia e } \\
\text { Antropologia }\end{array}$ & Ciências Sociais & Ciências Sociais & 17 \\
\hline Educação Especial & $\begin{array}{c}\text { Pedagogia, Terapia } \\
\text { Ocupacional e Fisioterapia }\end{array}$ & Educação & 48 \\
\hline Didática & Pedagogia & Educação & 16 \\
\hline Fonoaudiologia & Fonoaudiologia & Fonoaudiologia & 23 \\
\hline Filosofia & Filosofia & Filosofia & 17 \\
\hline $\begin{array}{l}\text { Ciências Políticas e } \\
\text { Econômicas }\end{array}$ & Relações Internacionais & $\begin{array}{c}\text { Relações } \\
\text { Internacionais }\end{array}$ & 18 \\
\hline $\begin{array}{l}\text { Administração e } \\
\text { Supervisão Escolar }\end{array}$ & Pedagogia & Educação & 11 \\
\hline Fonte: Faculdade de fil & &
\end{tabular}

Fonte: Faculdade de filosofia ciências (2010).

$\mathrm{Na}$ análise dos dados referente ao número de artigos publicados, foi possível observar que os docentes do Departamento de Ciências Políticas e Econômicas obtiveram o maior número de artigos publicados na plataforma Lattes $(P<0,05)$, em relação a outros departamentos. Entretanto, a maior parte de seus resultados foi publicada em artigos não indexados ao ISI e/ou SCOPUS. Essa característica também foi observada nos docentes pertencentes aos outros oito departamentos, ou seja, publicaram a maior parte dos seus trabalhos em artigos regionais e locais (Tabela 2).

Tabela 2 - Valor P do teste de hipótese entre os departamentos da FFC.

\begin{tabular}{|c|c|c|c|c|c|c|c|c|}
\hline & $\begin{array}{l}\text { Administra } \\
\text { ção e } \\
\text { Supervisão } \\
\text { Escolar }\end{array}$ & $\begin{array}{l}\text { Ciência } \\
\text { da } \\
\text { Informaç } \\
\text { ão }\end{array}$ & $\begin{array}{c}\text { Ciências } \\
\text { Políticas } \\
\text { e } \\
\text { Econômi } \\
\text { cas }\end{array}$ & $\begin{array}{c}\text { Didátic } \\
\text { a }\end{array}$ & $\begin{array}{l}\text { Educaç } \\
\text { ão } \\
\text { Especi } \\
\text { al }\end{array}$ & $\begin{array}{c}\text { Filoso } \\
\text { fia }\end{array}$ & $\begin{array}{c}\text { Fonoaudiolo } \\
\text { gia }\end{array}$ & $\begin{array}{c}\text { Psicologia } \\
\text { da } \\
\text { Educação }\end{array}$ \\
\hline \multicolumn{9}{|l|}{ Lattes } \\
\hline $\begin{array}{l}\text { Ciência da } \\
\text { Informação } \\
\text { Ciências }\end{array}$ & 1 & $x$ & 1 & 1 & 1 & 1 & 1 & 1 \\
\hline $\begin{array}{l}\text { Políticas e } \\
\text { Econômicas }\end{array}$ & 1 & 1 & $X$ & 0,462 & 0,215 & 0,510 & 1 & 1 \\
\hline Didática & 1 & 1 & 0,462 & $x$ & 1 & 1 & 1 & 1 \\
\hline $\begin{array}{l}\text { Educação } \\
\text { Especial }\end{array}$ & 1 & 1 & 0,215 & 1 & $x$ & 1 & 1 & 1 \\
\hline Filosofia & 1 & 1 & 0,510 & 1 & 1 & $x$ & 1 & 1 \\
\hline $\begin{array}{c}\text { Fonoaudiologi } \\
\mathrm{a}\end{array}$ & 1 & 1 & 1 & 1 & 1 & 1 & $x$ & 1 \\
\hline $\begin{array}{l}\text { Psicologia da } \\
\text { Educação }\end{array}$ & 1 & 1 & 1 & 1 & 1 & 1 & 1 & $x$ \\
\hline $\begin{array}{l}\text { Sociologia e } \\
\text { Antropologia }\end{array}$ & 1 & 1 & 1 & 1 & 1 & 1 & 1 & 1 \\
\hline $\begin{array}{c}\text { ISI } \\
\text { Ciência da } \\
\text { Informação }\end{array}$ & 1 & $x$ & 1 & 1 & 1 & 1 & 0,497 & 1 \\
\hline Ciências & 1 & 1 & $x$ & 1 & 1 & 1 & 0,367 & 1 \\
\hline
\end{tabular}


Políticas e

Econômicas

Didática

Educação

Especial

Filosofia

Fonoaudiologi

$$
\text { a }
$$

Psicologia da

Educação

Sociologia e

Antropologia

\section{SCOPUS}

Ciência da

Informação

Ciências

Políticas e

Econômicas

Didática

Educação

Especial

Filosofia

Fonoaudiologi

$$
\text { a }
$$

Psicologia da

Educação

Sociologia e

Antropologia

$\boldsymbol{h}$ pelo ISI

Ciência da

Informação

Ciências

Políticas e

Econômicas

Didática

Educação

Especial

Filosofia

Fonoaudiologi

$$
\text { a }
$$

Psicologia da

Educação

Sociologia e

Antropologia

\section{$h$ pelo}

\section{SCOPUS}

Ciência da

Informação

Ciências

Políticas e

Econômicas

Didática

Educação

Especial

Filosofia

Fonoaudiologi

$$
\text { a }
$$

Psicologia da

Educação

Sociologia e

Antropologia

1
1
1

0,236

$$
0,497
$$

0,367

1

$\begin{array}{lll}x & 1 & 1\end{array}$

0,103

0,153

0,166

0,103

0,153

0,166

$\mathrm{x}$

1

0,206

1

1

$x$

1

$\begin{array}{lll}1 & 1 & 1\end{array}$

1

1

1

$\begin{array}{ll}1 & 1\end{array}$

0,039

0,030

0,019

1

11

0,039

0,019

0,018

0,026

0,024

$\begin{array}{ccc}1 & 1 & X \\ 0,018 & 0,026 & 0,024\end{array}$

11

$\begin{array}{cccccccc}1 & 1 & 1 & 1 & 1 & 1 & 0,009 & 1 \\ 1 & x & 1 & 1 & 1 & 1 & 0,100 & 1 \\ 1 & 1 & x & 1 & 1 & 1 & 0,070 & 1 \\ 1 & 1 & 1 & x & 1 & 1 & 0,113 & 1 \\ 1 & 1 & 1 & 1 & x & 1 & 0,794 & 1 \\ 1 & 1 & 1 & 1 & 1 & x & 0,095 & 1 \\ 1 & 0,100 & 0,070 & 0,113 & 0,794 & 0,095 & x & 0,714 \\ 1 & 1 & 1 & 1 & 1 & 1 & 0,714 & x \\ & 1 & 1 & 1 & 1 & 1 & 0,095 & 1\end{array}$

1

\begin{tabular}{cccccccc}
1 & 1 & 1 & 1 & 1 & 1 & 0,009 & 1 \\
1 & $x$ & 1 & 1 & 1 & 1 & 0,100 & 1 \\
1 & 1 & $x$ & 1 & 1 & 1 & 0,070 & 1 \\
1 & 1 & 1 & $x$ & 1 & 1 & 0,113 & 1 \\
1 & 1 & 1 & 1 & $x$ & 1 & 0,794 & 1 \\
1 & 1 & 1 & 1 & 1 & $x$ & 0,095 & 1 \\
1 & 0,100 & 0,070 & 0,113 & 0,794 & 0,095 & $x$ & 0,714 \\
1 & 1 & 1 & 1 & 1 & 1 & 0,714 & $x$ \\
\hline 307 & 1 & 1 & 1 & 1 & 1 & 0,095 & 1
\end{tabular}

1

$\begin{array}{cccccccc}1 & 1 & 1 & 1 & 1 & 1 & 0,009 & 1 \\ 1 & x & 1 & 1 & 1 & 1 & 0,100 & 1 \\ 1 & 1 & x & 1 & 1 & 1 & 0,070 & 1 \\ 1 & 1 & 1 & x & 1 & 1 & 0,113 & 1 \\ 1 & 1 & 1 & 1 & x & 1 & 0,794 & 1 \\ 1 & 1 & 1 & 1 & 1 & x & 0,095 & 1 \\ 1 & 0,100 & 0,070 & 0,113 & 0,794 & 0,095 & x & 0,714 \\ 1 & 1 & 1 & 1 & 1 & 1 & 0,714 & x \\ & 1 & 1 & 1 & 1 & 1 & 0,095 & 1\end{array}$

11

$\mathrm{x} \quad 1$

Fonte: Faculdade de filosofia ciências (2010). "x" refere-se às comparações já realizadas. 
Os resultados demonstraram que o Departamento de Fonoaudiologia apresentou valores mais elevados para os números de artigos indexados no SCOPUS/ISI e apresentou o índice- $h$ pelo SCOPUS/ISI mais elevado $(P<0,05)$, quando comparados aos professores dos outros oito departamentos (Figura 1).

Figura 1 - Quantidade de artigos de periódicos publicados no CL, indexados ao ISI e o índice $h$ dos docentes pertencentes à FFC
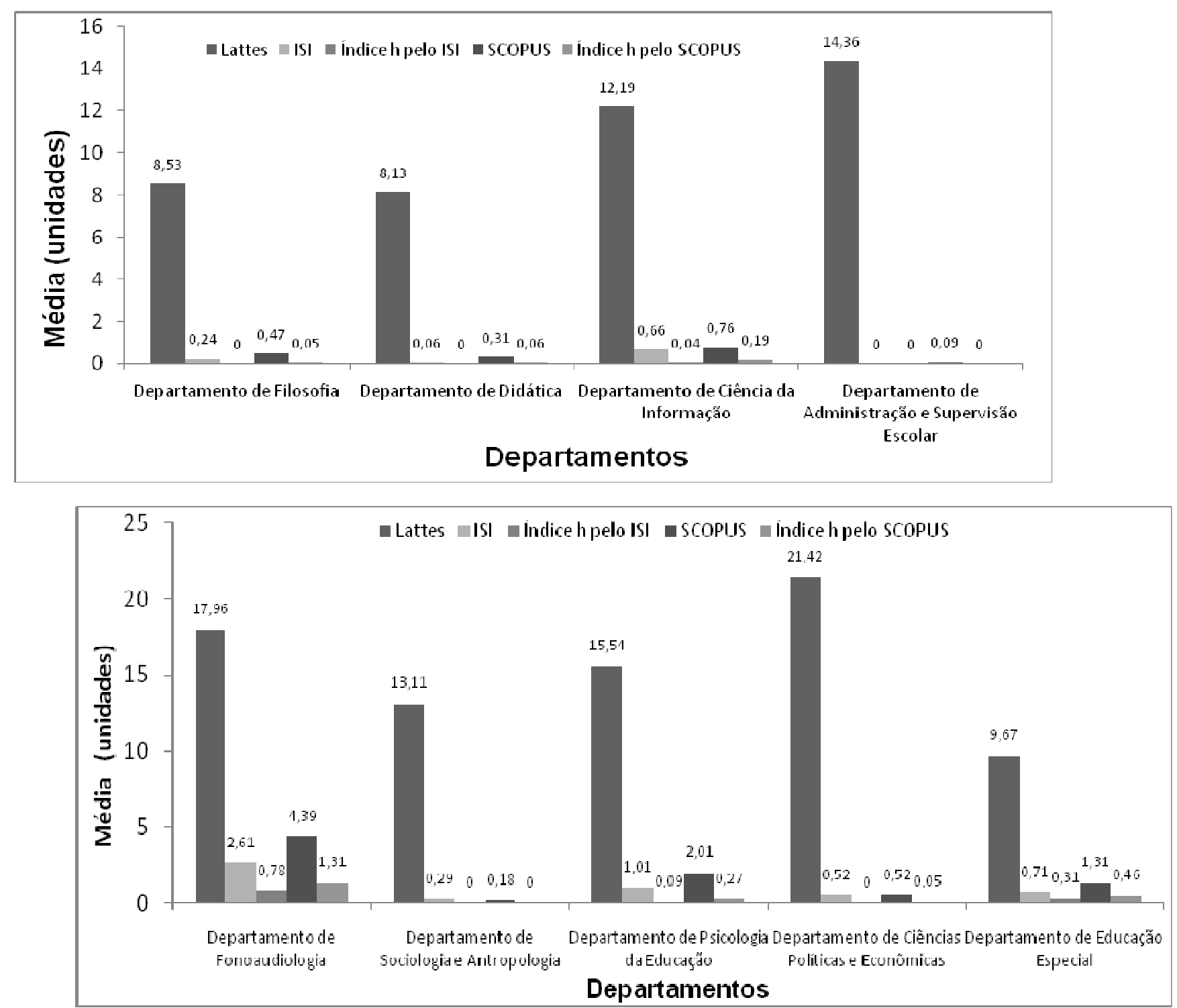

Fonte: Faculdade de filosofia ciências (2010).

Com relação à análise de dados relativa ao número de orientações em andamento e/ou concluídos dos docentes em Programas de PósGraduação, observamos que o departamento de Administração e Supervisão Escolar apresentou números mais elevados comparados aos outros departamentos, o que ocorre devido à consolidação do Programas de Pós-Graduação (PPG) em Educação, que possui conceito 5 pela CAPES (FACULDADE DE FILOSOFIA E CIÊNCIAS, 2012) e, além disso, o departamento possui apenas 11 docentes. Ao analisarmos as orientações 
em Programas de Pós-Graduação pelos docentes do Departamento de Fonoaudiologia, observamos médias baixas de orientações, o que pode ser explicado, pois o departamento não possuía programa de Pós-Graduação (Figura 2). Entretanto, em fevereiro de 2011, iniciou-se o PPG em Fonoaudiologia (FACULDADE DE FILOSOFIA E CIÊNCIAS, 2011), que possivelmente alavancará o número de orientações em Programas de PósGraduação do Departamento de Fonoaudiologia.

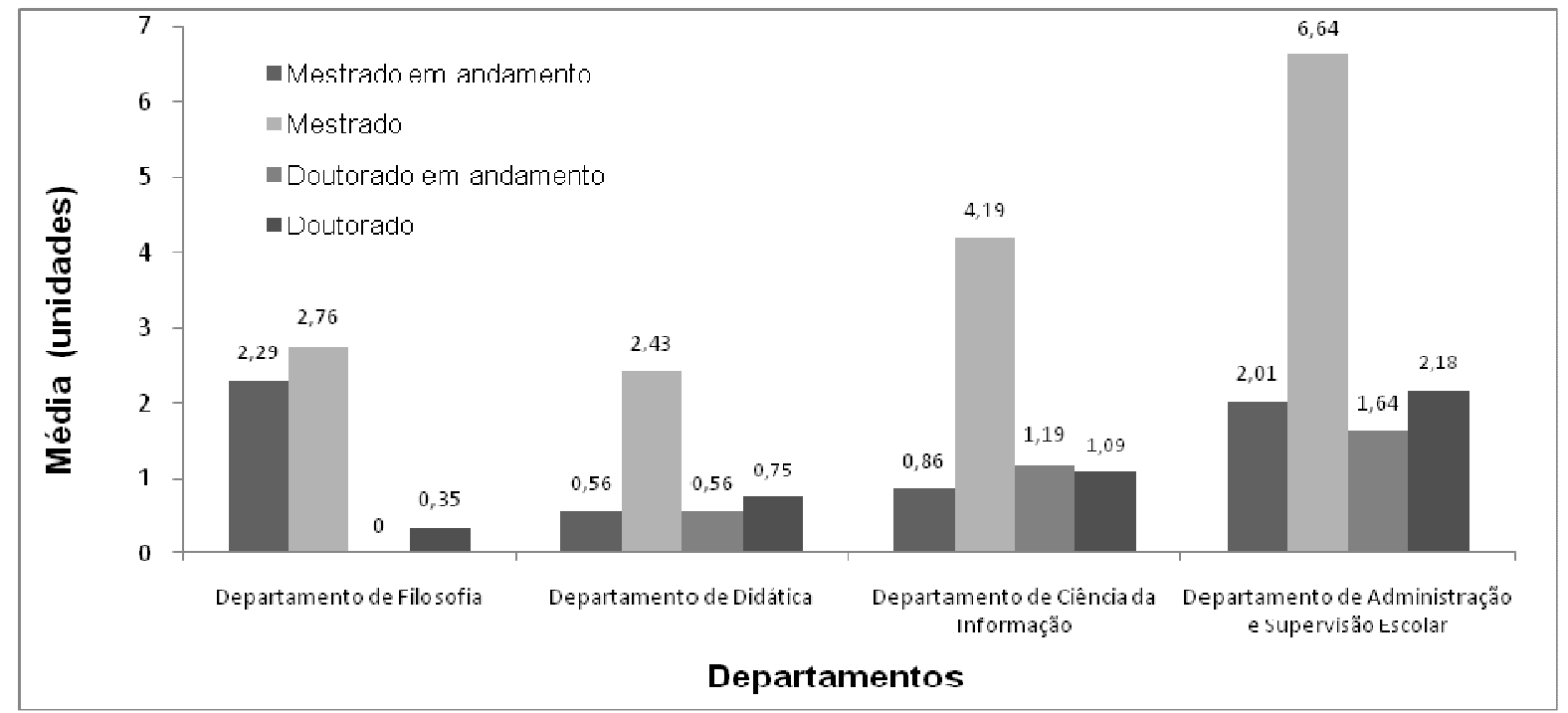

Figura 2 - Média de orientações em Programas de Pós-Graduação pelos

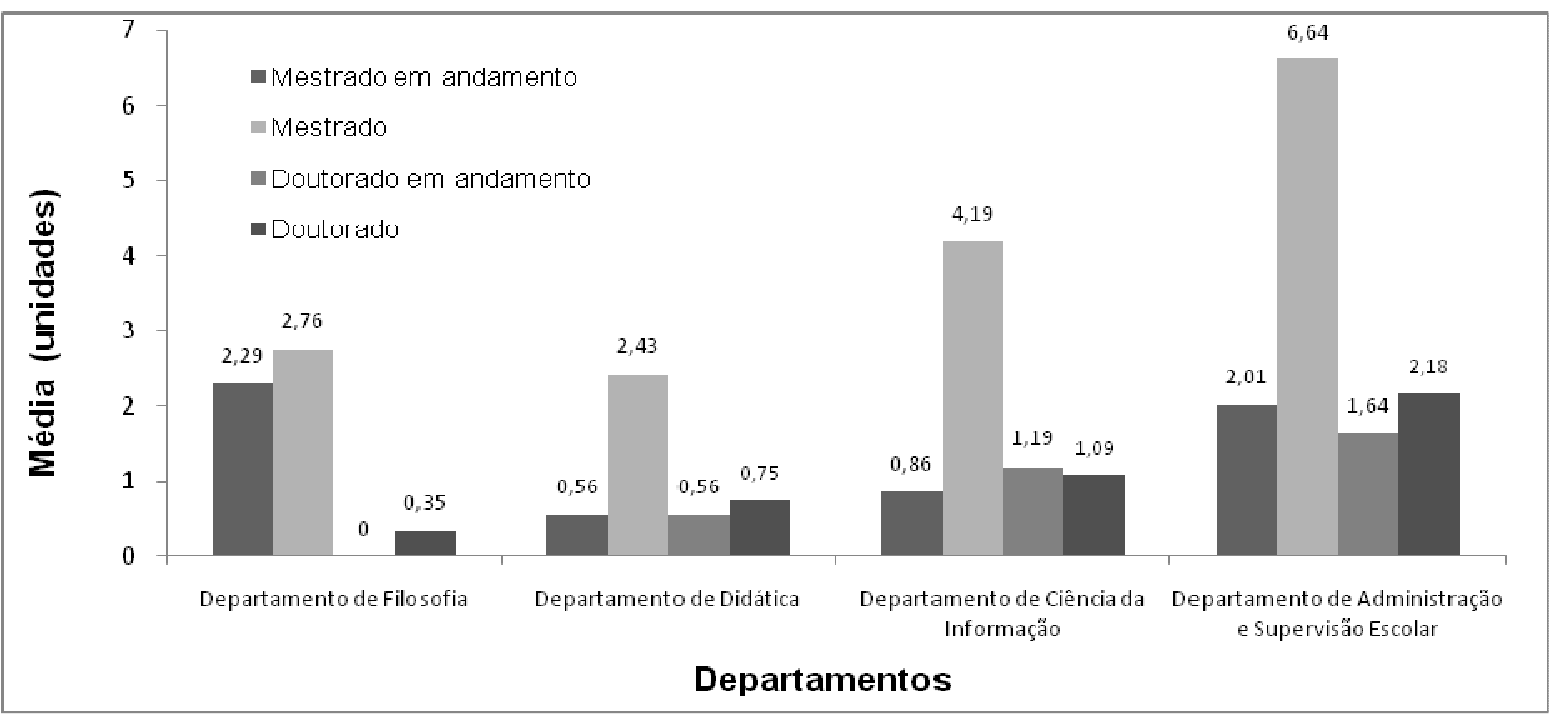

docentes dos nove departamentos da FFC-UNESP

Fonte: Faculdade de filosofia ciências (2010).

Para uma análise mais crítica dos dados, foi calculado a média de livros publicados e capítulos de livros pela Plataforma Lattes. Essa análise foi necessária devido à característica da área de Humanidades e Humanas, na qual é comum a publicação de livros e capítulos de livros. A tabela 3 apresenta a média dos livros publicados e capítulos de livros na plataforma Lattes. 
Tabela 3 - Média de livros e capítulos publicados, obtidos pela Plataforma Lattes

\begin{tabular}{lcc}
\hline \multicolumn{1}{c}{ Departamento } & Livros Publicados & Capítulos de Livros \\
\hline Psicologia da Educação & 2,09 & 5,55 \\
\hline Ciência da Informação & 2,52 & 11,01 \\
\hline Sociologia e & 2,41 & 5,35 \\
Antropologia & 1,25 & 5,48 \\
\hline Educação Especial & 2,25 & 6,63 \\
\hline Didática & 0,87 & 5,04 \\
\hline Fonoaudiologia & 2,12 & 4,71 \\
\hline Filosofia & 4,26 & 12,53 \\
\hline Ciências Políticas e & 4,91 & 9,18 \\
Econômicas & \multirow{2}{*}{ Administração e } & \\
Supervisão Escolar & &
\end{tabular}

Fonte: Faculdade de filosofia ciências (2010).

\section{Discussão}

A relação entre o financiamento e os rumos da pesquisa é registrada desde o século XVII. O próprio Galileu tinha de se comportar como cortesão em Florença, e a Academia Francesa de Ciência era estimulada a deixar as pesquisas curiosas e se dedicar às pesquisas úteis ao serviço do rei e do Estado. Dessa forma, o fomento científico, inicialmente de caráter privado e posteriormente público, não pode ser desmembrado da própria Revolução Científica (BURKE, 2003).

Atualmente, as agências de fomento nacionais, tais como Conselho Nacional de Desenvolvimento Científico e Tecnológico (CNPq), Coordenação de Aperfeiçoamento de Pessoal de Nível Superior (Capes) e Fundações de Amparo à Pesquisa (FAPs), nos diversos Estados, avaliam o pesquisador e as equipes de pesquisa através dos índices bibliométricos, dando peso maior às publicações em periódicos internacionais de grande impacto (LUIZ, 2003). Em contrapartida, as reduções das verbas têm criado um ambiente de trabalho estressante, levando o pesquisador à síndrome de esgotamento e ao sofrimento mental (De MEIS et al., 2003).

A Plataforma "Thompson ISI Web of Science" não indexa revistas de todas as áreas em cada País, apenas cobre, com a produção de países com ciência emergente, as lacunas deixadas na produção cientifica dos países centrais. Por exemplo, a Revista de Medicina Tropical.

Além disso, a produção cientifica na Pós-Graduação brasileira não é divulgada em revistas regionais e locais, pois o sistema de avaliação da Capes exige que o Corpo editorial ou Conselho editorial de revistas nacionais tenham representatividade nacional e internacional. Por esta razão, emite nota para os títulos de periódicos nacionais, os quais estão disponíveis no Sistema Qualis, na página da Capes (MINISTÉRIO DA EDUCAÇÃO, 2011).

Diferenças interessantes entre os professores da FFC vinculados aos nove departamentos foram encontradas nas análises de dados, mostrando que os índices de produtividade investigados, neste estudo, podem ser 
sensíveis ao campo da concentração. A análise dos departamentos mostrou que o Departamento de Ciências Políticas e Econômicas apresentou números elevados de artigos $(P<0,05)$ publicados na Plataforma Lattes $(21,42)$, quando comparados aos outros departamentos.

Como já ressaltado, a FFC possui seis programas de pós-graduação em Ciência da Informação, Ciências Sociais, Educação, Fonoaudiologia, Filosofia e Relações Internacionais e observamos resultados muito distintos em relação às orientações em Programas de Pós-Graduação nos diferentes departamentos. A baixa média de orientações em PPG no Departamento de Fonoaudiologia pode ser explicada pelo fato de não haver, até o mês de fevereiro de 2011, um programa de Pós-Graduação no Departamento.

Os departamentos na área de humanas/humanidades apresentaram médias mais baixas do índice- $h(P<0,05)$ e poucos artigos indexados pelo ISI e SCOPUS ( $\mathrm{P}<0,05)$, comparado ao Departamento de Fonoaudiologia. É possível inferir a existência de uma relação entre o número de artigos indexados pelo ISI e o índice- $h$ : quanto maior é o número de artigos indexados, no ISI, mais elevado é o índice- $h$. Assim, neste caso, a pequena porcentagem de artigos indexados pelo ISI dos departamentos da área de Humanas/Humanidades pode ser o fator responsável para o baixo índice- $h$. Outro fator a se considerar é a particularidade do campo de concentração (TORRO-ALVES et al., 2007). A maioria dos artigos de Educação, Sociologia, Filosofia e Biblioteconomia são publicados em revistas regionais, talvez porque suas pesquisas referem-se a assuntos do interesse local. Essas revistas locais geralmente não são indexadas pelo ISI e nem pelo SCOPUS e, assim, o impacto dos artigos publicados pelos professores da Pedagogia, Arquivologia, Biblioteconomia, Filosofia e Sociologia não podem ser apropriadamente avaliados com os dados coletados do Thompson ISI Web of Science ou SCOPUS.

Atualmente, a cobertura do ISI é ainda um problema grande, principalmente porque somente uma pequena porcentagem (5\%) dos artigos publicados no mundo está incluída neste banco de dados, ou seja, cerca de 7 mil títulos, dos quais apenas 81 são revistas brasileiras (SCOPUS-ELSEVIER, 2011). Recentemente, a "SCOPUS", que é o maior banco de dados de resumos e citações, com cerca de 17 mil revistas, surgiu para competir com Thompson ISI Web of Science, pois realiza funcionalidades semelhantes como: 1) controle de autoridade (author identifier); 2) análise de citações (citation tracker); e 3) cálculo do Índice Hirsch (índice- $h$ ). A "SCOPUS" conta com 29 milhões de resumos, 265 milhões de referências, 18 milhões de páginas sobre patentes e 265 milhões de páginas web indexadas por SCIRUS, além das tradicionais revistas científicas acadêmicas. Entretanto, no banco de dados do SCOPUS estão somente resumos de artigos publicados a partir de 1996, ou seja, artigos publicados antes desse ano não são contados. Recentemente, a SCOPUS incorporou o banco de dados SciELO à sua base e, desta forma, 
473 periódicos de 12 países ibero-americanos e 1 caribenho estarão indexados ao SCOPUS.

A análise do curriculum Lattes foi muito importante, porque mostra que o baixo índice- $h$ não está relacionado necessariamente a uma baixa produtividade científica, mas, sim, à pequena porcentagem de artigos indexados pelo ISI e SCOPUS.

\section{Conclusões}

Em resumo, o atual estudo indicou que a maioria dos departamentos da FFC-Unesp obtiveram resultados similares na avaliação do índice- $h$, número de artigos indexados pelo ISI e SCOPUS. Entretanto, em relação aos artigos no currículo Lattes, a maior média $(21,42)$ foi encontrada pelo Departamento de Ciências Políticas e Econômicas. A respeito do índice- $h$ e artigos indexados no ISI/SCOPUS, os valores mais baixos encontrados ( $P$ $<$ 0,05) dos departamentos de Psicologia da Educação, Ciência da Informação, Sociologia e Antropologia, Educação Especial, Didática, Filosofia, Ciências Políticas e Econômicas e Administração e Supervisão Escolar pode ser associado às características do campo da concentração e da cobertura limitada do banco de dados ISI e SCOPUS. Finalmente, estes resultados indicam que $o$ índice- $h$ é muito sensível ao campo de concentração e não deve ser usado como o único fator de avaliação da produção científica de pesquisa de um indivíduo. Assim, o emprego da Plataforma Lattes apresenta outros conteúdos que podem ser considerados para mensurar a produtividade científica de tais pesquisadores.

\section{Referências}

BRAGA, G. M. Informação, ciência, política científica: o pensamento de Derek de Solla Price. Ciência da Informação, v. 3, n. 2, p. 155-177, 1974.

BURKE, P. Uma história social do conhecimento de Gutenberg a Diderot. Rio de Janeiro: Jorge Zahar Editor, 2003.

CASTRO, C. M. Ciência e universidade. Rio de Janeiro: Zahar, 1985. pp. 56.

De MEIS, L. et al. The growing competition in Brazilian science: rites of passage, stress and burnout. Brazilian Journal of Medical and Biological Research, v. 36, p. 1135-1141, 2003.

FACULDADE DE FILOSOFIA E CIÊNCIAS. Universidade Estadual Paulista. Apresentação. 2010. Disponível em: <http://www.marilia.unesp.br>. Acesso em: 10 fev. 2010.

FACULDADE DE FILOSOFIA E CIÊNCIAS. Programa de Pós-Graduação em Fonoaudiologia. Apresentação. 2011. Disponível em: <http://www.marilia.unesp.br/index.php?CodigoMenu=7007\&CodigoOpcao=7007>

Acesso em: 10 fev. 2010. 
FACULDADE DE FILOSOFIA E CIÊNCIAS. Apresentação do Programa de Pós-Graduação em Educação. 2012. Disponível em: <http://www.marilia.unesp.br/posedu/>. Acesso em: 10 fev. 2010.

GARFIELD, E. Quantitative analysis of the scientific literature and its implications for science policymakingin latin america and the caribbean. Bulletin of the PanAmerican Health Organization, v. 29, p. 87-95,1995.

GLÂNZEL, W. On the $h$-index: a mathematical approach to a new measure of publication activity and citation impact. Scientometrics, v. 67, p. 315321, 2006.

HERCULANO, R. D. et al. Produção científica na Faculdade de Filosofia, Ciências e Letras de Ribeirão Preto - Universidade de São Paulo: aplicação do índice de Hirsch. Medicina (Ribeirão Preto), v. 41, n. 3, p. 343-350, 2008.

HERCULANO, R. D.; NORBERTO, A. M. Q. Uma análise percentual dos periódicos publicados pelos docentes de fonoaudiologia da Universidade São Paulo. Medicina (Ribeirão Preto), v. 42, n. 3, p. 308-314, 2009 a.

HERCULANO, R. D; NORBERTO, A. M. Q. Comparison of scientific bibliographic productivity in undergraduate courses of speech-language and hearing science at Universidade of São Paulo using the Hirsh's Index. Journal of Applied Sciences, v. 9, p. 4095-4098, 2009b.

HIRSCH, J. E. An index to quantify an individual's scientific research output. Proc. Natl. Acad. Sci., USA, v. 102, p. 16569-16572, 2005.

IRVINE, J.; MARTIN, B. R. International comparisons of scientific performance revisited. Scientometrics, v. 15, n. 5-6, p.369-392, 1989.

LUIZ, A. J. B. Importância de textos em português para a apropriação do conhecimento em ciência e tecnologia no Brasil. Cadernos de Ciência \& Tecnologia, v. 20, n. 3, p. 543-554, 2003.

MACIAS-CHAPULA, C A. O papel da informetria e da cienciometria e sua perspectiva nacional e internacional. Ciência da Informação, v. 27, n. 2, p. 134-140, 1998.

MERTON, R. K. Social and democratic social structure. In: SOCIAL theory and social structure. New York: Free Press, 1957. p. 550-561.

MINISTÉRIO DA EDUCAÇÃO. WebQualis-QUALIS-CAPES/MEC: consulta e classificação de periódicos. 2011. Disponível em: <http://qualis.capes.gov.br/webqualis/>. Acesso em: 10 fev. 2010.

OKUBO, Y. Bibliometric indicators and analysis of research systems: methods and examples. Paris: OCDE/GD, 1997.

PACKER, A. L.; MENEGHINI, R. Articles with authors affiliated to Brazilian institutions published from 1994 to 2003 with 100 or more citations: I the weight of international collaboration and the role of the networks. $A n$. Acad. Bras. Cienc, v. 78, n. 4, p. 841-853, 2006. 
PLATAFORMA LATTES. Conselho Nacional de Desenvolvimento Científico e Tecnológico (CNPq). Plataforma Lattes. 2011. Disponível em: <http://buscatextual.cnpq.br/buscatextual/index.jsp >. Acesso em: 20 Jan. 2011.

PRICE, D. J. Networks of Scientific Papers. Science, v. 149, n. 3683, p. 510-515, 1965.

PRICE, D. J. Measuring the size of science. Proceedings of the Israel Academy of Sciences and Humanities, v. 4, p. 98-106, 1969.

PRICE D. J. O desenvolvimento da cidadania: análises histórica, filosófica, sociológica e econômica. Rio de Janeiro: Livros Teóricos e Científicos, 1976.

ROEDIGER, H. L. The h-index in science: a new measure of scholarly contribution. Observer, v. 19, n. 4, 2006. Disponível em: $<$ http://www.psychologicalscience.org/observer/getArticle.cfm?id=1971>. Acesso em: 20 Jan. 2011.

SCIENTIFIC ELECTRONIC LIBRARY ONLINE (SciELO). 2011. Disponível em: <http:// www.scielo.org>. Acesso em: 31 Jan. 2011.

SCOPUS-ELSEVIER. 2011. Disponível em: <http:// www.scopus.com>. Acesso em: 30 Jan. 2011.

SPINAK, E. Diccionario enciclopédico de bibliometría, cienciometría e informetría. Montevideo: [s. n.], 2006.

STREHL, L.; SANTOS, C. A. dos. Indicadores de qualidade da atividade científica. Ciência Hoje, v. 31, n. 186, p. 34-39, 2002.

TORRO-ALVES, N. et al. Hirsch's index: a case study conducted at the Faculdade de Filosofia, Ciências e Letras de Ribeirão Preto, Universidade de São Paulo. Braz J Med Biol Res, v. 40, pp. 1529-1536, 2007.

THOMPSON ISI WEB of SCIENCE. 2011. Disponível em: $<$ http://portal.isiknowledge.com/portal.cgi?DestApp=WOS\&Func=Frame. $>$. Acesso em: 5 fev. 2011.

VANTI, N. Análise cienciométrica de um banco eletrônico de dissertações e teses na área de antropologia. In: CONGRESSO BRASILEIRO DE BIBLIOTECONOMIA E DOCUMENTAÇÃO (CBBD), 19., Porto Alegre, 2000. Anais... Brasília: FEBAB, 2000, p. 1-28. Disponível em <http://dici.ibict.br/archive/00000791/>. Acesso em: 1 dez. 2011.

VELHO, L. A. Avaliação do desempenho cientifico. Cadernos USP, São Paulo, n. 1, p. 22-40, 1986.

VESSURI, H. M. C. La Revista Científica Periférica: el caso de Acta Científica Venezolana. Interciencia, v. 12, n. 3, p. 124-34, 1987. 